Research Article

\title{
Theoretical Analysis of the Faraday Effect in Carbon Nanotubes with Arbitrary Chirality
}

\author{
Abbas Zarifi \\ Department of Physics, Yasouj University, Yasouj, Iran \\ Correspondence should be addressed to Abbas Zarifi; zarifi@mail.yu.ac.ir
}

Received 9 March 2013; Accepted 4 April 2013

Academic Editors: S. Bud'ko, H. Hibino, and J. Tempere

Copyright ( 2013 Abbas Zarifi. This is an open access article distributed under the Creative Commons Attribution License, which permits unrestricted use, distribution, and reproduction in any medium, provided the original work is properly cited.

\begin{abstract}
Using tight-binding model with nearest neighbour interactions, the optical properties of carbon nanotubes under the influence of an external magnetic field are analyzed. First, dipole matrix elements for two cases of light polarized parallel as well as perpendicular to the nanotube axis are analyzed. A close form analytic expression for dipole matrix is obtained for carbon nanotubes with arbitrary chirality in the case of light polarized parallel to the nanotube axis. Then the diagonal and off-diagonal elements of the frequencydependent susceptibility in the presence of an axial magnetic field are investigated. The off-diagonal elements are applied to calculate the interband Faraday rotation and the Verdet constant. These effects should be clearly detectable under realistic conditions using weak magnetic fields.
\end{abstract}

\section{Introduction}

The rotation of the polarization of a plane-polarized electromagnetic wave passing through a substance under the influence of a static magnetic field along the direction of propagation is known as the Faraday rotation $[1,2]$. It arises because right circular polarization and left circular polarization waves propagate with different phase velocities, and a phase difference results between the two waves. For a weak absorption and then ignoring the extinction coefficient, the wave vector components $\left(k_{+}\right)$of a linearly polarized light have different refractive indices $n_{+}$, and then the rotation of the polarization plane known as optical Faraday rotation is proportional to the difference in the refractive indices, $n_{+}-n_{-}$. The Faraday rotation for solids, liquids, and gases has been investigated both theoretically and experimentally [28]. Optical Faraday rotation near the resonance regime has been proposed to measure a single-electron spin in a single quantum dot inside a microcavity [9]. Numerical calculations have been made [10] to calculate the Faraday rotation in graphite by extrapolation from high-field tight binding calculations. It has been demonstrated that the graphene turns the polarization by several degrees in modest magnetic fields [11]. Experimentally, Faraday rotation is measured mainly under nonresonant conditions, that is, for frequencies far from resonance, where the absorption is weak. In this case, the angle of rotation is proportional to the imaginary part of the off-diagonal elements of susceptibility. By studying the offdiagonal elements of susceptibility, the goal of this paper is to investigate the Faraday rotation in single-walled carbon nanotubes (SWCNs) threaded by a parallel magnetic field. Hence, we calculate the off-diagonal elements of susceptibility in the presence of magnetic field. For a weak magnetic field and a nonoptically active solid, one can treat the Faraday effect as a first-order effect in $B$. We will therefore concentrate on the low magnetic field limit to expand the off-diagonal elements of the susceptibility tensor to the first order in the magnetic field and calculate the Faraday rotation as a first-order effect in $B$. While the diagonal elements of the susceptibility are quite insensitive to even huge magnetic fields, rather modest fields lead to detectable changes in the off-diagonal response. In particular, we compute the Verdet constant of CNs in aqueous dispersions and show that under realistic conditions, the $\mathrm{CN}$ signature should be clearly detectable above the background.

This paper is organized as follows. In Section 2, we present a general reduced form of the Hamiltonian for $\mathrm{CNs}$ in the presence of an external magnetic field and analyze the corresponding eigenvalues and eigenvectors. In Section 3, we 
discuss the dipole matrix elements for two cases of light polarized parallel as well as perpendicular to the nanotube axis, and then obtain diagonal and off-diagonal elements of the optical susceptibility tensor in the presence of a magnetic field. Expanding the off-diagonal elements to the first order in the magnetic field, we obtain the Faraday rotation and the Verdet constant for CNs in Section 4. Finally, a summary is given in Section 5.

\section{Tight-Binding Framework}

First of all, we study the electronic structure of CNs denoted by $(n, m)$ notation in the presence of an external magnetic field. In our old paper [12], based on tight-binding approximation, we used a four by four Hamiltonian matrix for zigzag CNs and did some calculations to provide analytic expressions for the off-diagonal elements of the linear susceptibility in the presence of magnetic field and then we studied the Faraday effect in zigzag CNs. In this paper, we intend to use a two by two Hamiltonian matrix instead for all different kinds of CNs which is more convenient and study the Faraday effect in CNs with arbitrary chirality. Considering only nearestneighbour interactions, the nonzero Hamiltonian elements $H_{B A}^{(B)}(\vec{k})=H_{A B}^{(B)^{*}}(\vec{k})$ in the presence of an axial external magnetic field are given by $[12,13]$

$$
H_{A B}^{(B)}(k)=\gamma_{0} \sum_{l=1}^{3} \exp \left(i \frac{e}{\hbar} \Delta_{l A}(t)\right) \exp \left(-i \vec{k} \cdot \vec{b}_{l}^{A}\right)
$$

where $\gamma_{0} \sum_{l} \exp \left(-i \vec{k} \cdot \vec{b}_{l}^{A}\right)$ is known as the Hamiltonian matrix elements for zero magnetic field, $\gamma_{0} \approx 2.89 \mathrm{eV}$ is the nearest neighbor overlap integral, $\vec{b}_{l}^{A}(l=1,2,3)$ are the nearest neighbor carbon atom vectors from $A$ to $B$ sites, and $e>0$ is the elementary charge. Moreover, $\Delta_{l A}(t)$ is given by [12]

$$
\Delta_{l A}(t)=\int_{0}^{1} \vec{b}_{l}^{A} \cdot \vec{A}\left(\vec{R}_{0 A}+\vec{b}_{l}^{A}(\eta-1)\right) d \eta .
$$

Here, $\vec{R}_{0 A}$ is the position vector of $A$ carbon atom in the 0 th unit cell, and $\vec{A}$ is the vector potential associated with the magnetic field. The magnetic field $\vec{B}=B \widehat{z}$ is along the nanotube axis, and the symmetric gauge $\vec{A}(\vec{r})=$ $B / 2(-y, x, 0)$ is applied. Therefore, one can obtain the phase factor associated with the magnetic field as follows:

$$
\Delta_{l A}(t)=\frac{B}{2}\left\{b_{l y}^{A} X_{0 A}-b_{l x}^{A} Y_{0 A}\right\}
$$

Taking $A$ atom at the origin as $A_{0}$, the phase factor for its three nearest neighbour $B$ atoms is given by [13]

$$
\begin{gathered}
\Delta_{1 A_{0}}(t)=\frac{B R^{2} \theta_{1}}{2}, \quad \Delta_{2 A_{0}}(t)=-\frac{B R^{2} \theta_{2}}{2}, \\
\Delta_{3 A_{0}}(t)=-\frac{B R^{2} \theta_{3}}{2} .
\end{gathered}
$$

Here, $R$ is the radius of a $\mathrm{CN}$ and because of the smallness of angles, the approximations $\sin \left(\theta_{i}\right)=\theta_{i}$ are made, and $\theta_{i}$ s as the associated angles of the three nearest neighbour atoms are given by [13]

$$
\begin{gathered}
\theta_{1}=\frac{\pi(n+m)}{n^{2}+m^{2}+n m}, \quad \theta_{2}=\frac{\pi m}{n^{2}+m^{2}+n m}, \\
\theta_{3}=\frac{\pi n}{n^{2}+m^{2}+n m} .
\end{gathered}
$$

Introducing the above equations into (1), one has

$$
\begin{gathered}
H_{A B}^{(B)}(\vec{k})=\gamma_{0}\left\{e^{-i k_{x} b} e^{i e B R^{2} \theta_{1} /(2 \hbar)}+e^{i k_{x} b / 2-i k_{y} b \sqrt{3} / 2} e^{-i e B R^{2} \theta_{2} /(2 \hbar)}\right. \\
\left.+e^{i k_{x} b / 2+i k_{y} b \sqrt{3} / 2} e^{-i e B R^{2} \theta_{3} /(2 \hbar)}\right\},
\end{gathered}
$$

where $b=a_{C-C}=a / \sqrt{3}$ is the bound length of grapheme, and the wave vector components are defined by

$$
\begin{aligned}
& k_{x}=K_{1} \cos (\alpha)-K_{2} \sin (\alpha), \\
& k_{y}=K_{1} \sin (\alpha)+K_{2} \cos (\alpha),
\end{aligned}
$$

where $K_{1}^{\mu} L=2 \pi \mu, \mu=0, \ldots, N-1$ are quantized wave vectors in the circumferential direction parallel to the chiral vector $\vec{L},-\pi / T \leq K_{2} \leq \pi / T$ is a longitudinal continuous wave vector parallel to the translational vector $\vec{T}$, and $\alpha=\pi / 6-\cos ^{-1}\left[(2 n+m) /\left(2 \sqrt{n^{2}+m^{2}+n m}\right)\right]$. Solving the Hamiltonian equation, the energy eigenvalues are given by

$$
\begin{aligned}
E_{c, v}^{(B)}(k) & = \pm E^{(B)}(k) \\
& = \pm \gamma_{0} \sqrt{3+2 \cos \left(\delta_{1}\right)+2 \cos \left(\delta_{2}\right)+2 \cos \left(\delta_{3}\right)}
\end{aligned}
$$

Here,

$$
\begin{aligned}
& \delta_{1}=\frac{b}{2}\left(3 k_{x}-\sqrt{3} k_{y}\right)-\frac{B R^{2} e\left(\theta_{1}+\theta_{2}\right)}{2 \hbar}, \\
& \delta_{2}=\sqrt{3} b k_{y}+\frac{B R^{2} e\left(\theta_{2}-\theta_{3}\right)}{2 \hbar}, \\
& \delta_{3}=\frac{b}{2}\left(3 k_{x}+\sqrt{3} k_{y}\right)-\frac{B R^{2} e\left(\theta_{1}+\theta_{3}\right)}{2 \hbar} .
\end{aligned}
$$

The similar results have previously been obtained by others [14-16]. The obtained eigenvalues demonstrate a metalsemiconductor transition depending on the magnitude of the field [16]. Increasing the magnitude of the field leads to an increased band gap in metallic CNs and a reduced band gap for semiconductor CNs for a limited range of magnetic field. However, the changes will not always be smooth but show a periodic behavior. Using $E_{c}^{(B)}(k)$ and 
$E_{v}^{(B)}(k)$ to solve the eigenvector problem, the corresponding normalized eigenvectors are given by

$$
\begin{gathered}
\vec{v}(k)=\left(C_{v A}, C_{v B}\right)=\frac{1}{\sqrt{2}}\{-g(k), 1\}, \\
\vec{c}(k)=\left(C_{c A}, C_{c B}\right)=\frac{1}{\sqrt{2}}\{g(k), 1\}, \\
g(k)=\frac{H_{A B}^{(B)}(\vec{k})}{E^{(B)}(k)} .
\end{gathered}
$$

Previously, it was shown that orbital overlap is essential for a correct description of off-diagonal magneto-optical effects in graphite as well as zigzag CNs $[11,12]$. The energy dispersion relation for the above Hamiltonian matrix, including the overlap matrix, is given by [11]

$$
E_{c, v}(k)=\frac{E_{c, v}^{(B)}(k)+\varepsilon_{2 p}}{1-E_{c, v}^{(B)}(k) s_{0} / \gamma_{0}}
$$

where $\varepsilon_{2 p}=-5 \mathrm{eV}$ is the on-site energy, $s_{0}=0.1$ is the nearest-neighbor overlap integral [10], and the eigenvectors are the same as those of the Hamiltonian matrix without considering the overlap. As mentioned in [12], by including the overlap matrix, the symmetry between valence and conduction bands is removed and then leads to the compression of the valence bands and the expansion of the conduction bands.

\section{Magneto-Optical Properties}

We take the constant magnetic field along the $z$ direction (the direction of the nanotube axis) and first calculate the dipole moment elements for two cases of light polarized parallel as well as perpendicular to the nanotube axis. Hence, through (14) in [13] as a general equation, the dipole moment elements are given by

$$
\begin{aligned}
\vec{d}_{c \vec{k}^{\prime}, v \vec{k}}= & \frac{-e \gamma_{0}}{N E_{c v}\left(\vec{k}^{\prime}, \vec{k}\right)} \\
& \times\left\{C_{c A}^{*}\left(\vec{k}^{\prime}\right) C_{v B}(\vec{k}) \sum_{s} e^{i\left(\vec{k}-\vec{k}^{\prime}\right) \cdot \vec{R}_{s A} \vec{v}^{A}(\vec{k})}\right. \\
& \left.\quad+C_{c B}^{*}\left(\vec{k}^{\prime}\right) C_{v A}(\vec{k}) \sum_{s} e^{i\left(\vec{k}-\vec{k}^{\prime}\right) \cdot \vec{R}_{s B}} \vec{v}^{B}(\vec{k})\right\} .
\end{aligned}
$$

Here, $N$ is the number of unit cells, $E_{c v}\left(\vec{k}^{\prime}, \vec{k}\right)=E_{c}^{(B)}\left(\vec{k}^{\prime}\right)-$ $E_{v}^{(B)}(\vec{k}), \vec{v}^{A, B}$ atomic dipole vectors defined by (13) in [13] are independent of magnetic fields, and $C_{c A, B}\left(C_{v A, B}\right)$ are magnetic-dependent elements of conduction and valence eigenvectors for two atoms $A$ and $B$, respectively, defined by (10). In the case of parallel polarization, all $A$ atoms in different cells have the same component of the atomic dipole

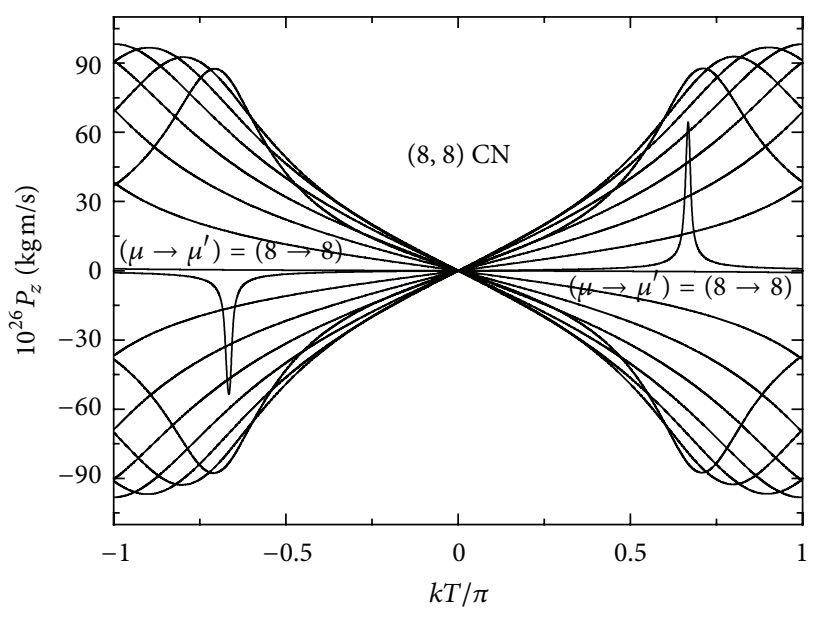

FIGURE 1: $k$-dependence of the long-axis momentum matrix elements $P_{z}$ for a $(8,8) \mathrm{CN}$. The plot is for $B=100 \mathrm{~T}$.

vector and similarly for all $B$ atoms. Therefore, the axial $(z)$ component of the dipole matrix element is given by

$$
d_{c v}^{z}(k)=\frac{-e}{E_{c v}(k)} \sum_{A, B}^{2} c_{A}^{*}(k) v_{B}(k) \frac{1}{i} \frac{d H_{A B}^{(B)}}{d k}
$$

In analogy with (6) in [17], the $z$ component of the dipole matrix element for all SWCNs is given by

$$
\begin{aligned}
d_{c v}^{z}(k)= & \frac{2 e a \gamma_{0}^{2}}{\sqrt{3} E_{c v}^{2}(k)} \\
& \times\left[\sin (\alpha)\left\{\cos \left(2 X_{2}\right)-\cos \left(X_{2}\right) \cos \left(X_{1}\right)\right\}\right. \\
& \left.\quad+\sqrt{3} \cos (\alpha) \sin \left(X_{2}\right) \sin \left(X_{1}\right)\right]
\end{aligned}
$$

where

$$
\begin{aligned}
& X_{1}=\frac{1}{2}\left(3 b k_{x}-\frac{B R^{2} e\left(2 \theta_{1}+\theta_{2}+\theta_{3}\right)}{2 \hbar}\right), \\
& X_{2}=\frac{1}{2}\left(\sqrt{3} b k_{y}+\frac{B R^{2} e\left(\theta_{2}-\theta_{3}\right)}{2 \hbar}\right) .
\end{aligned}
$$

Obtaining a general analytical form for dipole matrix elements in the presence of magnetic field is one of the most interesting results obtained in this paper. It is shown that the dipole moment vanishes for transitions between bands with different $\mu$. Figure 1 shows the $z$ component of the momentum $\left(P_{z}\right)$ matrix element for a $(8,8) \mathrm{CN}$, where $P_{z}=\left(m_{e} / \hbar e\right) E_{l l} d_{c_{1} v_{l}}^{z}$ and the magnetic field $B=100 \mathrm{~T}$. In comparison with our plots in the absence of magnetic field, two sharp peaks have been created for subband transitions of $(8,8)$ in the presence of magnetic field. By means of equations (18a) and (18b) in [13], the $x$ component of electric dipole 


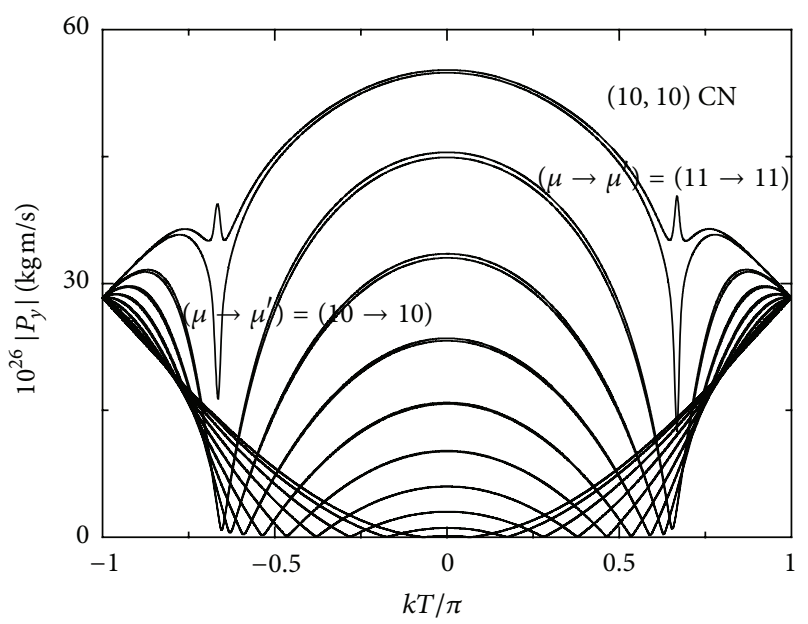

FIgURE 2: $k$-dependence of the short-axis momentum matrix elements $P_{y}$ for a $(10,10) \mathrm{CN}$. The plot is for $B=100 \mathrm{~T}$.

vectors for light polarized perpendicular to the nanotube axis is given by

$$
\begin{aligned}
d_{c \vec{k}^{\prime}, \vec{k}}^{x}= & \frac{-e \gamma_{0} \delta_{\vec{K}_{2} \vec{k}_{2}^{\prime}}}{2 \sqrt{2} E_{c v}\left(\vec{k}^{\prime}, \vec{k}\right)} \\
& \times\left[\left\{C_{c A}^{*}\left(\vec{k}^{\prime}\right) v_{+}^{A_{0}}(\vec{k})-C_{v A}(\vec{k}) v_{+}^{B_{0}}(\vec{k})\right\} \delta_{\mu^{\prime}=\mu-1}\right. \\
& \left.+\left\{C_{c A}^{*}\left(\vec{k}^{\prime}\right) v_{-}^{A_{0}}(\vec{k})-C_{v A}(\vec{k}) v_{-}^{B_{0}}(\vec{k})\right\} \delta_{\mu^{\prime}=\mu+1}\right] .
\end{aligned}
$$

Here,

$$
\begin{aligned}
v_{ \pm}^{A_{0}}=R & \left\{e^{-i k_{x} b}\left(1-e^{ \pm i \theta_{1}}\right)\right. \\
+ & e^{i k_{x} b / 2-i k_{y} b \sqrt{3} / 2}\left(1-e^{\mp i \theta_{2}}\right) \\
& \left.+e^{i k_{x} b / 2+i k_{y} b \sqrt{3} / 2}\left(1-e^{\mp i \theta_{3}}\right)\right\}, \\
v_{ \pm}^{B_{0}}=-v_{ \pm}^{A_{0} *} & \\
=-R & \left\{e^{i k_{x} b}\left(1-e^{\mp i \theta_{1}}\right)\right. \\
& +e^{-i k_{x} b / 2+i k_{y} b \sqrt{3} / 2}\left(1-e^{ \pm i \theta_{2}}\right) \\
& \left.+e^{-i k_{x} b / 2-i k_{y} b \sqrt{3} / 2}\left(1-e^{ \pm i \theta_{3}}\right)\right\} .
\end{aligned}
$$

For the $y$ component of electric dipole, the following relations are established. For $\mu^{\prime}=\mu+1$ case, $d_{c \vec{k}, v \vec{k}^{\prime}}^{y}=-i d_{c \vec{c}, v \vec{k}^{\prime}}^{x}$ and for $\mu^{\prime}=\mu-1$ case, $d_{c \vec{k}, v \vec{k}^{\prime}}^{y}=i d_{c \vec{k}, v \vec{k}^{\prime}}^{x}$. Figure 2 shows the $y$ component of the absolute value of the momentum $\left(P_{y}\right)$ matrix element for a $(10,10) \mathrm{CN}$, where $P_{x}=\left(m_{e} / \hbar e\right) E_{l l} d_{c_{1} v_{l}}^{x}$ and the magnetic field $B=100 \mathrm{~T}$. The results of (14) and (16) are in full agreement with similar equations in [18].

To discuss the diagonal as well as off-diagonal susceptibility, we start with the general form of the linear susceptibility defined by [12]

$$
\chi_{i j}(\omega)=\frac{1}{\pi \varepsilon_{0} \sigma} \sum_{c, v} \int_{-\pi / T}^{\pi / T}\left[\frac{d_{v \vec{k}, \vec{k}^{\prime}}^{i} d_{c \vec{k}^{\prime}, v \vec{k}}^{j}}{E_{c \vec{k}^{\prime}, v \vec{k}}-\hbar \Omega}+\frac{d_{\vec{v},, \vec{k}^{\prime}}^{j} d_{c \vec{k}^{\prime}, v \vec{k}}^{i}}{E_{c \overrightarrow{k^{\prime}}, v \vec{k}}+\hbar \Omega}\right] d k .
$$

Here, $d_{c \vec{k}^{\prime}, v \vec{k}}^{i}$ are dipole moment elements for a transition between valence band $v$ and conduction band $c$ at wave vectors $k$ and $k^{\prime}$, the nanotube cross-sectional area $\sigma=$ $\pi R^{2}, \varepsilon_{0}$ is the vacuum permittivity, $T$ is the length of the unit cell, and $\Omega=\omega+i \Gamma$ contains the photon frequency $\omega$ and the broadening parameter $\Gamma$. It is found that the diagonal susceptibility $\left(\chi_{z z}\right)$ is quite insensitive to the magnetic perturbation. Now we try to obtain the off-diagonal elements of susceptibility tensor. The off-diagonal susceptibility elements are zero in the absence of magnetic field. However, magnetic field leads to the nonzero off-diagonal elements which could be used to study the Faraday effect in SWCNs. Using equations (18a) and (18b) in [13] and relations between them, (18) can be written as

$$
\begin{aligned}
& \chi_{x y}(\omega, B) \\
& =\frac{i \hbar \Omega e^{2} \gamma_{0}^{2}}{\varepsilon_{0} L^{2}} \delta_{\vec{K}_{2} \vec{K}_{2}^{\prime}} \\
& \quad \times \sum_{c, v} \int_{-\pi / T}^{\pi / T}\left[\frac{\left|d_{c \vec{k}^{\prime}, \vec{k}}^{x+}\right|^{2}}{E_{c \vec{k}^{\prime}, v \vec{k}}^{2}\left(E_{c \vec{k}^{\prime}, v \vec{k}}^{2}-\hbar^{2} \Omega^{2}\right)} \delta_{\mu^{\prime}=\mu+1}\right. \\
& \left.\quad-\frac{\left|d_{c \overrightarrow{k^{\prime}}, v \vec{k}}^{x-}\right|^{2}}{E_{c \vec{k}^{\prime}, \vec{k}}^{2}\left(E_{c \vec{k}^{\prime}, v \vec{k}}^{2}-\hbar^{2} \Omega^{2}\right)} \delta_{\mu^{\prime}=\mu-1}\right] d k,
\end{aligned}
$$

where

$$
\begin{aligned}
& d_{c \vec{k}^{\prime}, \vec{k}}^{x+}=C_{c A}^{*}\left(\vec{k}^{\prime}\right) v_{+}^{A_{0}}(\vec{k})-C_{v A}(\vec{k}) v_{+}^{B_{0}}(\vec{k}), \\
& d_{c \vec{k}^{\prime}, v \vec{k}}^{x-}=C_{c A}^{*}\left(\vec{k}^{\prime}\right) v_{-}^{A_{0}}(\vec{k})-C_{v A}(\vec{k}) v_{-}^{B_{0}}(\vec{k}) .
\end{aligned}
$$

Inserting the eigenvector equations and atomic dipole vectors into (19), we get the off-diagonal elements of susceptibility for SWCNs with arbitrary chirality in the presence of a magnetic field.

\section{Linear Order of Magnetic Field}

As mentioned in our earlier calculations for zigzag CNs, when the external magnetic field $B$ is weak, we can treat the Faraday effect as a first-order effect in $B$. We will therefore restrict our calculations to weak magnetic fields. 


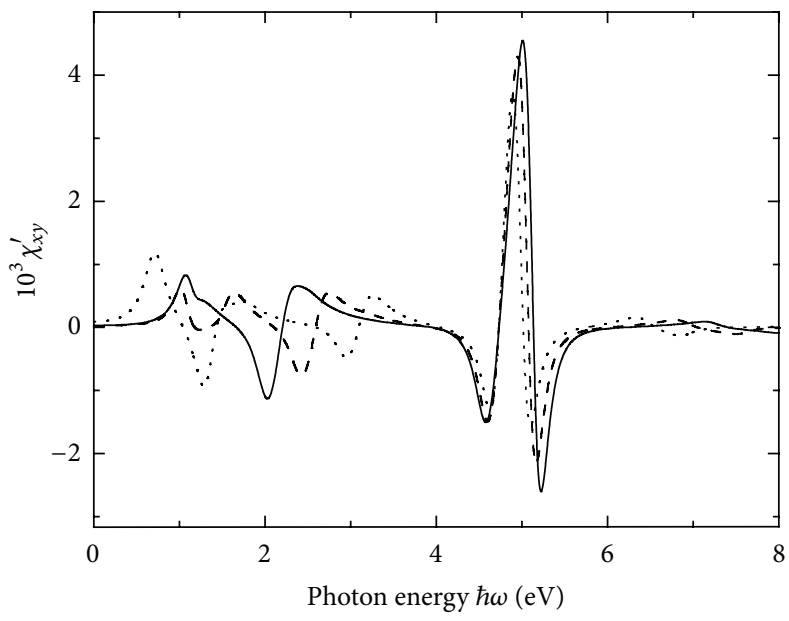

We ignore the relatively weak field dependence of the terms $d_{c \vec{k}^{\prime}, v \vec{k}}^{x+}$, and $d_{c \vec{k}^{\prime}, v \vec{k}}^{x-}$ and then expand the denominator of (19) to the first order in $B$ and express the susceptibility as $\chi_{x y}(\omega, B)=\left(\chi^{(+)}(\omega)-\chi^{(-)}(\omega)\right) \times B$. Moreover, it can be shown that $\chi^{(+)}(\omega)=-\chi^{(-)}(\omega)$, and therefore one can write $\chi_{x y}(\omega, B)=2 \chi^{(+)}(\omega) \times B$. After some simplifications, the fieldindependent coefficient $\chi^{(+)}(\omega)$ is given by

$$
\begin{aligned}
& \chi^{(+)}(\omega) \\
& =\frac{2 i \hbar \Omega e^{2} \gamma_{0}^{2}}{\varepsilon_{0} L^{2}} \delta_{\vec{K}_{2} \vec{K}_{2}^{\prime}} \\
& \times \sum_{c, v} \int_{-\pi / T}^{\pi / T}\left[\frac{\left|d_{c \vec{c}^{\prime}, v \vec{k}}^{x+}\right|^{2}\left(2 E_{c \vec{k}^{\prime}, v \vec{k}}^{2}-\hbar^{2} \Omega^{2}\right) E_{c \vec{k}^{\prime}, v \vec{k}}^{\prime}}{E_{c \vec{k}^{\prime}, v \vec{k}}^{3}\left(E_{c \vec{k}^{\prime}, v \vec{k}}^{2}-\hbar^{2} \Omega^{2}\right)^{2}} \delta_{\mu^{\prime}=\mu+1}\right] d k,
\end{aligned}
$$

where $E_{c \vec{k}^{\prime}, v \vec{k}}^{\prime}$ can be found by differentiated from (11) and then insert $B=0$, is given by

$$
\begin{aligned}
& E_{c \vec{k}^{\prime}, \vec{k}}^{\prime} \\
& \quad=E_{c}^{\prime}\left(k^{\prime}\right)-E_{v}^{\prime}(k) \\
& \quad=\left(1+\frac{s \varepsilon_{2 \pi}}{\gamma_{0}}\right)\left(\frac{E^{\prime}\left(k^{\prime}\right)}{\left(1-s / \gamma_{0} E\left(k^{\prime}\right)\right)^{2}}-\frac{E^{\prime}(k)}{\left(1-s / \gamma_{0} E(k)\right)^{2}}\right),
\end{aligned}
$$

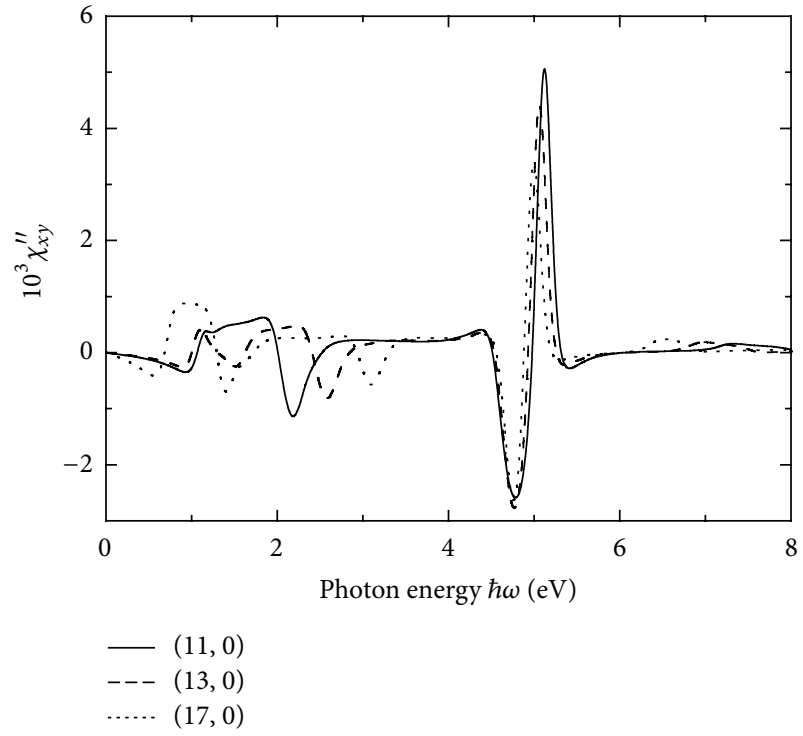

(b)

where

$$
\begin{aligned}
E^{\prime}(k)=\frac{-e R^{2} \gamma_{0}^{2}}{2 \hbar E(k)} & \\
\quad & \quad\left[\left(\theta_{1}+\theta_{2}\right) \sin \left[\frac{b}{2}\left(3 k_{x}-\sqrt{3} k_{y}\right)\right]\right. \\
& \quad+\left(\theta_{3}-\theta_{2}\right) \sin \left[\sqrt{3} b k_{y}\right] \\
& \left.+\left(\theta_{1}+\theta_{3}\right) \sin \left[\frac{b}{2}\left(3 k_{x}+\sqrt{3} k_{y}\right)\right]\right] .
\end{aligned}
$$

In the same manner for $E^{\prime}\left(k^{\prime}\right)$, the wave vector $k$ is inserted by $k^{\prime}$, where $E\left(k, k^{\prime}\right)=E^{(B=0)}\left(k, k^{\prime}\right)$.

No analytic calculation has been obtained for (21); however, in the form given, the integral is easily computed for all SWCNs numerically. Real $\left(\chi_{x y}^{\prime}\right)$ and imaginary $\left(\chi_{x y}^{\prime \prime}\right)$ parts of the off-diagonal elements of the susceptibility tensor for $(11,0),(13,0)$, and $(17,0) \mathrm{CNs}$ are shown in Figure 3. A very prominent resonance peak is observed for semiconductor zigzag CNs with odd $n$.

The off-diagonal elements of the susceptibility tensor for $(10,0),(14,0)$, and $(20,0)$ zigzag CNs are shown in Figure 4, and those for CNs with arbitrary chirality including $(6,4)$, $(8,6)$, and $(10,6)$ CNs are shown in Figure 5.

The behaviour of CNs with arbitrary chirality is almost similar to zigzag CNs with $n$ even. Although each $\mathrm{CN}$ shows some resonance peaks for real as well as imaginary parts of the off-diagonal susceptibility, the peak positions are different for different CNs. Therefore in an ensemble of CNs we expect that the dominant resonance peaks are related to zigzag CNs with $n$ odd. As mentioned in our earlier paper, 


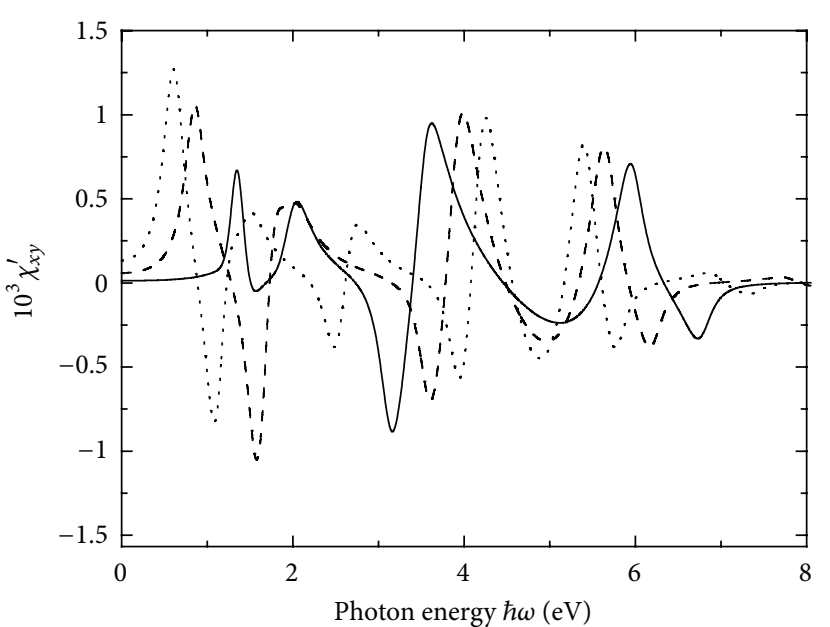

(a)

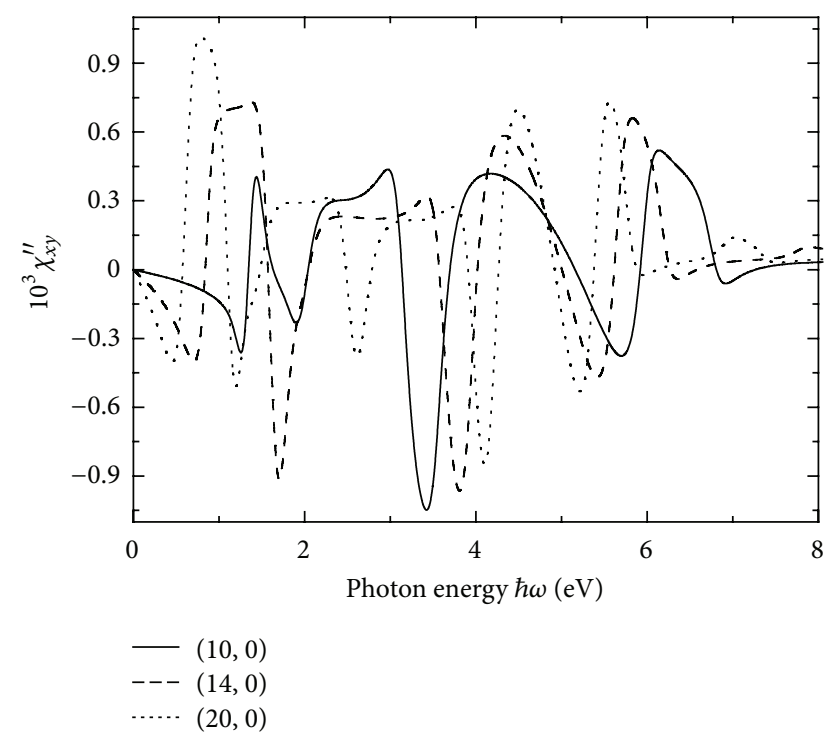

(b)

FIGURE 4: Real $\left(\chi_{x y}^{\prime}\right)$ and imaginary $\left(\chi_{x y}^{\prime \prime}\right)$ parts of the off-diagonal susceptibility for a magnetic field $B=1$ T for some zigzag CNs with even $n$.

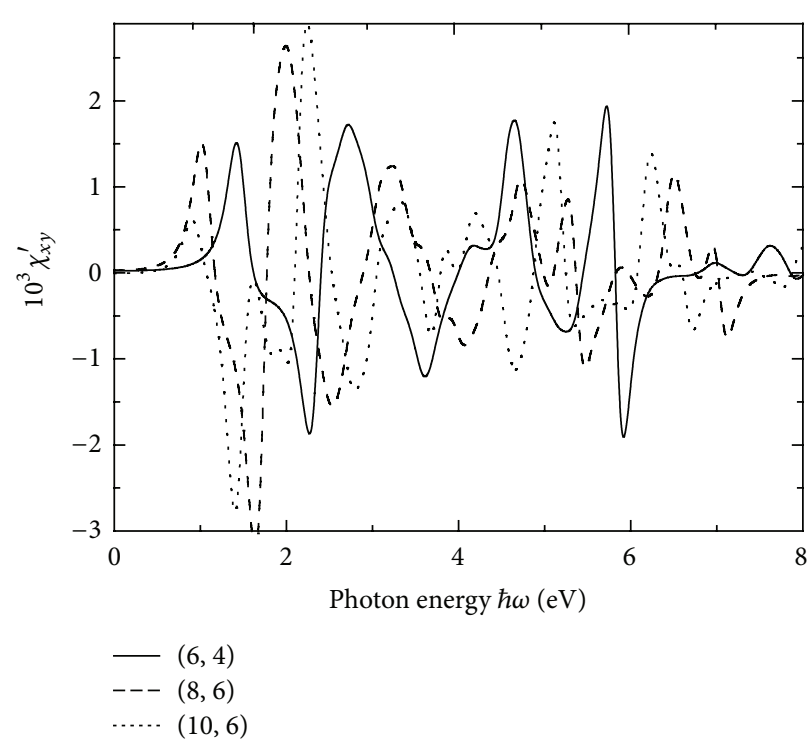

(a)

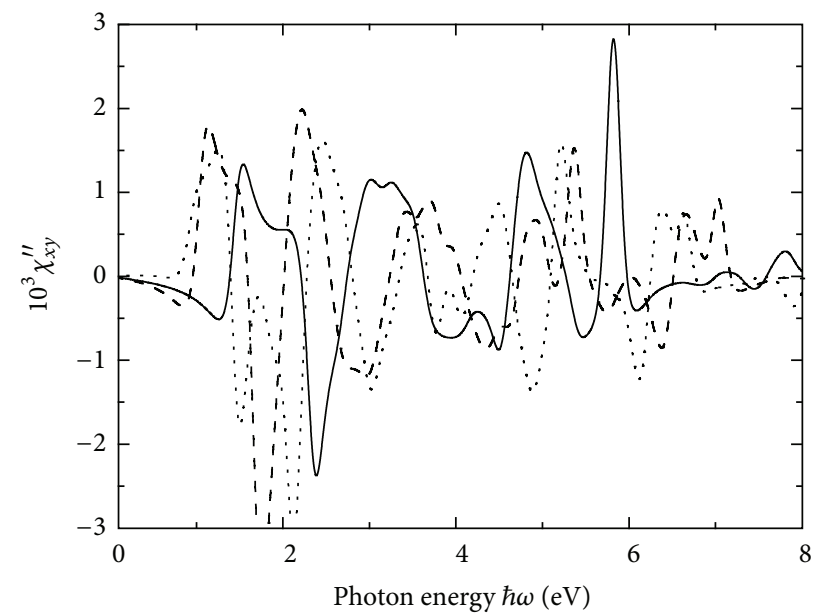

(b)

FIgURE 5: Real $\left(\chi_{x y}^{\prime}\right)$ and imaginary $\left(\chi_{x y}^{\prime \prime}\right)$ parts of the off-diagonal susceptibility for a magnetic field $B=1 \mathrm{~T}$ for some chiral CNs.

the plots are obtained using a rather large broadening of $\hbar \Gamma=0.15 \mathrm{eV}$ [12], which corresponds to relatively disordered samples. Using higher quality samples will only increase visibility.

Now we use the off-diagonal susceptibility to find the Faraday rotation and the Verdet constant. We consider aligned CNs suspended in a solution, and then the Faraday rotation $\phi$ at low magnetic fields is given by [12]

$$
\phi=\frac{\pi d}{\lambda} \frac{\chi_{x y}^{\prime \prime}}{n^{\prime}}
$$

where $\lambda$ is the wavelength, $d$ is the path length, and $n^{\prime}$ is the refractive index of the suspension. In our earlier calculations of investigating Faraday rotation for zigzag CNs, we considered water as suspension and then took $n^{\prime} \simeq$ 1.3 , and the absorption coefficient of the suspension was ignored. For simplicity, we choose the same suspension and then subsequently calculate the Verdet constant $V$ via the relation $\phi=V B d$. Hence, the Verdet constant $V$ is defined by $V=\left(2 \pi / \lambda n^{\prime}\right) \operatorname{Im} \chi^{(+)}(\omega)$. The energy dispersion of the Verdet constant for different semiconductor $\mathrm{CNs}$ threaded by a parallel magnetic field is plotted in Figure 6 . The Verdet 

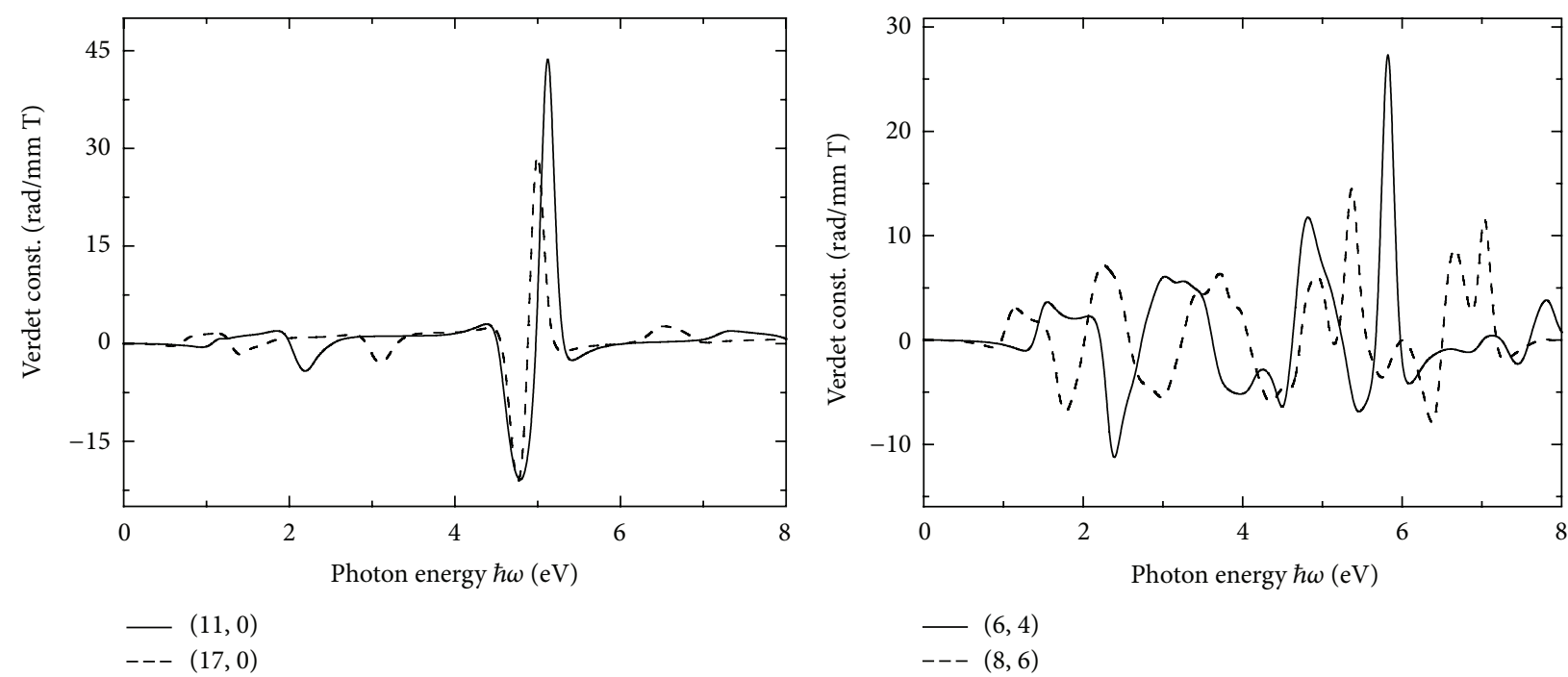

(a)

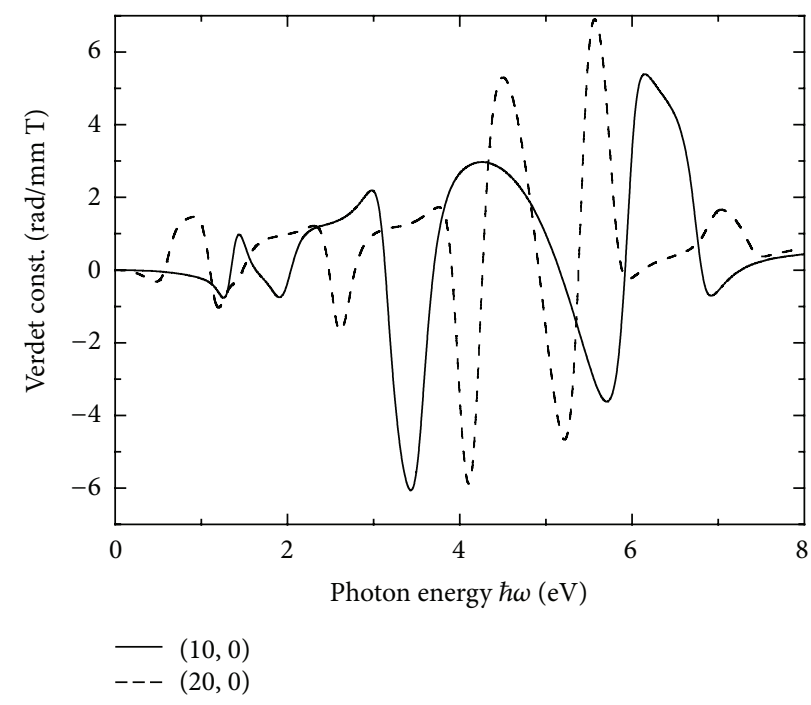

(b)

(c)

Figure 6: Dispersion of the Verdet constant for different semiconducting CNs. (a) shows odd $n$ zigzag CNs, (b) chiral CNs, and (c) even $n$ zigzag CNs.

constant for semiconductor zigzag CNs with odd $n$ exhibits a very prominent resonance at $\hbar \omega \simeq 5 \mathrm{eV}$. But for CNs with arbitrary chirality and zigzag $\mathrm{CNs}$ with even $n$, some resonances at different positions with different energies are found. Figure 7 demonstrates the Verdet constant related to a few of CNs. By including more CNs, only the peaks related to Zigzag CNs with odd $n$ happen at the same energy. Therefore, since averaging over an ensemble of CNs would normally tend to blur individual resonances, the prominent structures related to Zigzag CNs with odd $n$ are expected to prevail for ensembles containing many different semiconducting CNs.

\section{Summary}

A theoretical investigation based on a nonorthogonal tightbinding description has been made to study the Faraday effect in CNs. First dipole matrix elements of CNs have been calculated under the influence of a magnetic field. A close form analytic expression has been obtained in the case of light polarized parallel to the nanotube axis. Then the linear susceptibility of CNs has been investigated. The diagonal elements show no changes under the influence of an external magnetic field. The off-diagonal elements of the susceptibility tensor under the influence of an axial external magnetic field have been calculated semianalytically and expanded to the first order in the magnetic field. We have applied the obtained expressions to find the Faraday rotation and the Verdet constant of CNs for arbitrary chirality. Although Verdet constant in different $\mathrm{CNs}$ shows resonances at different energy positions, a very prominent resonance for all semiconductor zigzag CNs with odd $n$ has been 


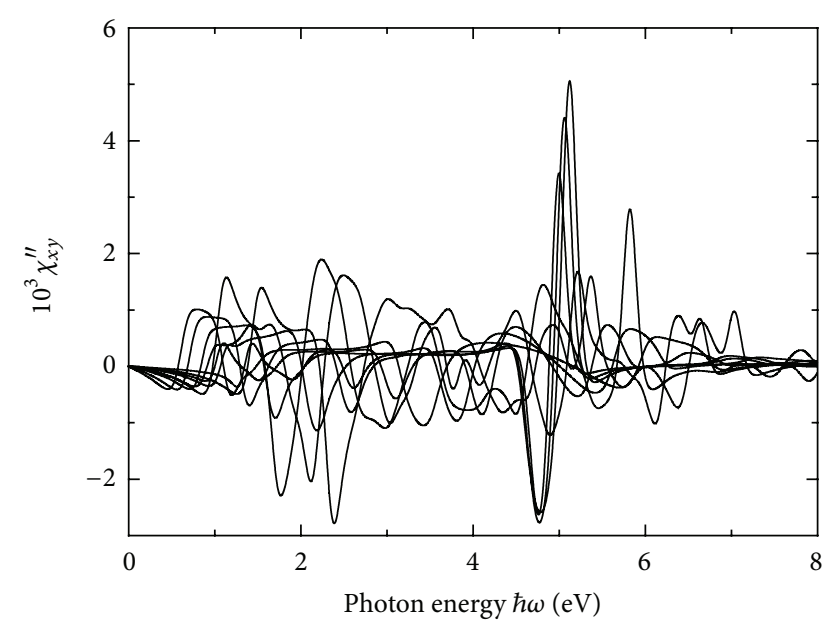

FIgURE 7: Dispersion of the Verdet constant for different groups of semiconducting CNs. The prominent peak is shown around $5 \mathrm{eV}$, which is for zigzag CNs with odd $n$.

demonstrated at $\hbar \omega \simeq 5 \mathrm{eV}$, which is expected to prevail for ensembles containing many different CNs.

\section{Acknowledgment}

The author is grateful to Professor S. V. Goupalov (Department of Physics, Jackson State University, USA) for devoting his time to read the paper and give some useful comments.

\section{References}

[1] M. Combescot and O. B. Matibet, "Faraday rotation in photoexcited semiconductors: A composite- exciton many body effect," Physical Review B, vol. 74, Article ID 125316, 2006.

[2] M. Barthélémy and D. J. Bergman, "Faraday effect in composites," Physical Review B, vol. 58, pp. 12770-12781, 1998.

[3] L. M. Roth, "Theory of the Faraday effect in solids," Physical Review, vol. 133, pp. A542-A553, 1964.

[4] H. S. Bennett and E. A. Stern, "Faraday effect in solids," Physical Review, vol. 137, pp. A448-A461, 1965.

[5] M. Balkanski, E. Amzallag, and D. Langer, "Interband Faraday rotation of II-VI compounds," Journal of Physics and Chemistry of Solids, vol. 27, no. 2, pp. 299-308, 1966.

[6] A. V. Kimel, Y. U. S. Grushko, A. G. Selitskii, and A. I. Sokolov, "Faraday effect in solutions of fullerene C60," Journal of Optical Technology, vol. 66, no. 8, p. 745, 1999.

[7] Y. Ruan, R. A. Jarvis, A. V. Rode, S. Madden, and B. L. Davies, "Wavelength dispersion of Verdet constants in chalcogenide glasses for magneto-optical waveguide devices," Optics Communications, vol. 252, pp. 39-45, 2005.

[8] W. A. Parkinson, S. P. A. Sauer, J. Oddershede, and D. M. Bishop, "Calculation of the Verdet constants for $\mathrm{H}_{2}, \mathrm{~N}_{2}$, CO, and $\mathrm{FH}$," The Journal of Chemical Physics, vol. 98, no. 1, pp. 487-495, 1993.

[9] C. Y. Hu, A. Young, J. L. O’Brien, W. J. Munro, and J. G. Rarity, "Giant optical Faraday rotation induced by a single-electron spin in a quantum dot: applications to entangling remote spins via a single photon," Physical Review B, vol. 78, Article ID 085307, 5 pages, 2008.
[10] T. G. Pedersen, "Tight-binding theory of Faraday rotation in graphite," Physical Review B, vol. 68, Article ID 245104, 6 pages, 2003.

[11] I. Crassee, J. Levallois, A. L. Walter et al., "Giant Faraday rotation in single- and multilayer graphene," Nature Physics, vol. 7, no. 1, pp. 48-51, 2011.

[12] A. Zarifi and T. G. Pedersen, "Theoretical analysis of the Faraday effect in zigzag carbon nanotubes," Physical Review B, vol. 77, Article ID 085409, 6 pages, 2008.

[13] A. Zarifi and T. G. Pedersen, "Universal analytic expression of electric-dipole matrix elements for carbon nanotubes," Physical Review B, vol. 80, Article ID 195422, 7 pages, 2009.

[14] J. P. Lu, "Novel magnetic properties of carbon nanotubes," Physical Review Letters, vol. 74, no. 7, pp. 1123-1126, 1995.

[15] R. Saito G, Dresselhaus, and M. S. Dresselhaus, Physical Properties of Carbon Nanotubes, Imperial College Press, London, UK, 2003.

[16] S. Roche, G. Dresselhaus, M. S. Dresselhaus, and R. Saito, "Aharonov-bohm spectral features and coherence lengths in carbon nanotubes," Physical Review B, vol. 62, no. 23, pp. 1609216099, 2000.

[17] A. Zarifi and T. G. Pedersen, "Linear optical and quadratic electro-optic response of carbon nanotubes: universal analytic expressions for arbitrary chirality," Journal of Physics, vol. 20, no. 27, Article ID 275211, 2008.

[18] S. V. Goupalov, A. Zarifi, and T. G. Pedersen, "Calculation of optical matrix elements in carbon nanotubes," Physical Review $B$, vol. 81, Article ID 153402, 4 pages, 2010. 

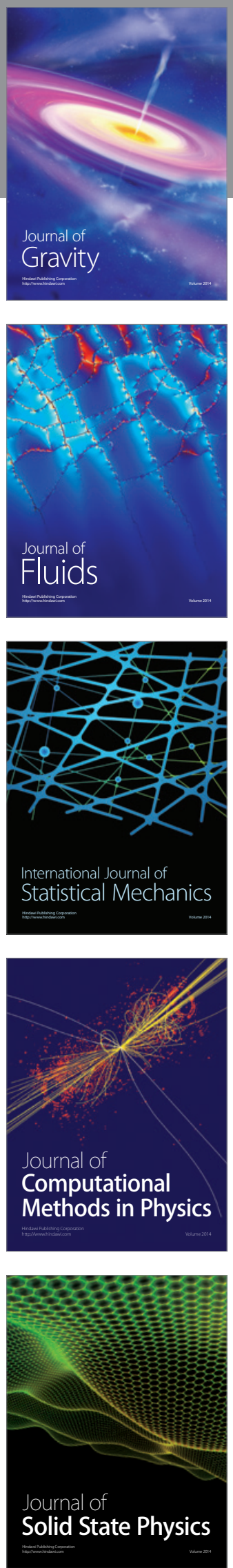

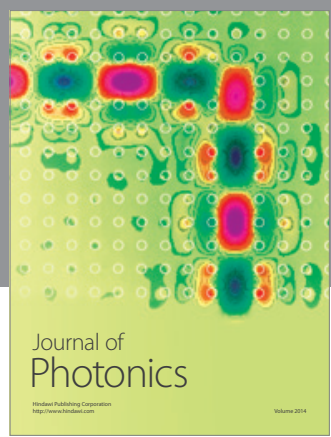

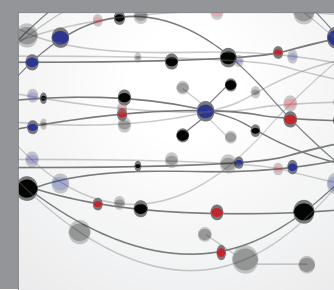

The Scientific World Journal

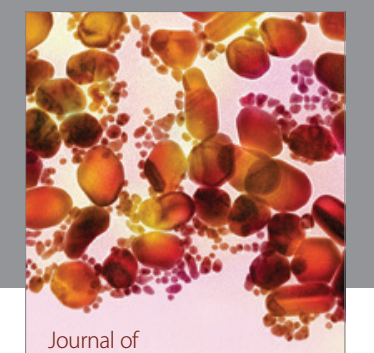

Soft Matter
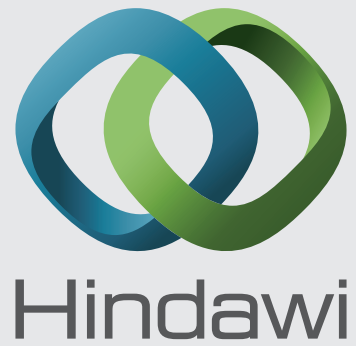

Submit your manuscripts at

http://www.hindawi.com
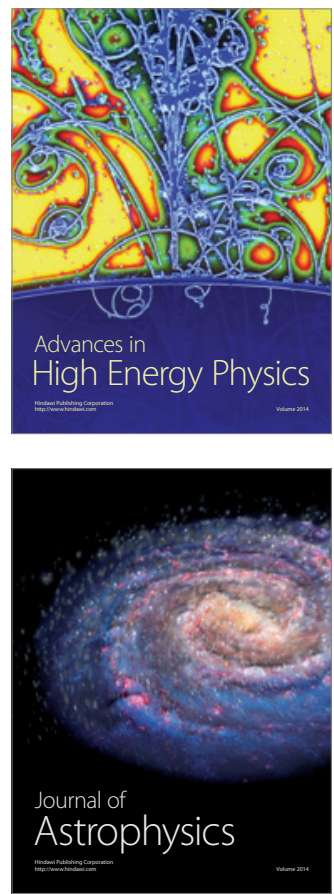
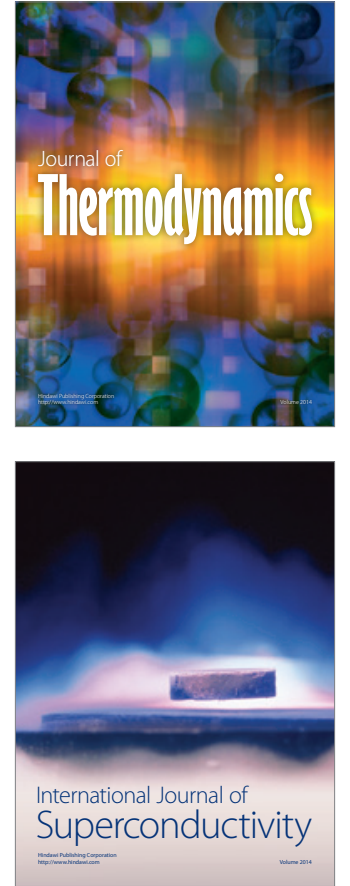
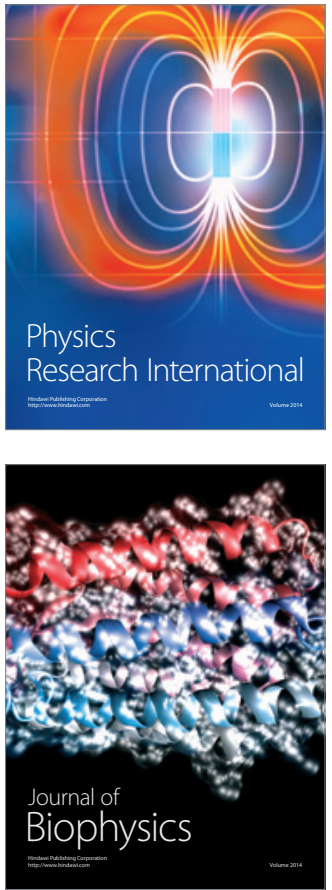
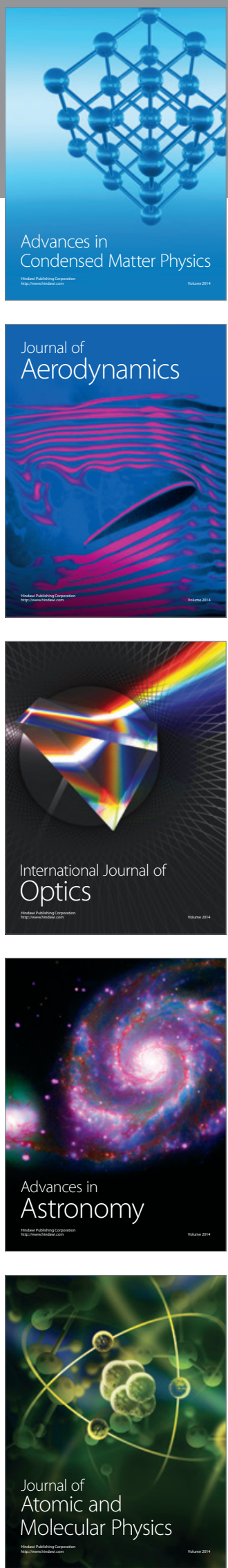